\title{
Dynamic Task Assignment in a Multiagent/Multitask Environment based on Module Conflict Resolution
}

\author{
Eiji Uchibe, Tatsunori Kato, Minoru Asada, Koh Hosoda \\ Graduate School of Eng., Dept. of Adaptive Machine Systems \\ Osaka University, Suita, Osaka 565-0871, Japan \\ uchibe@er.ams.eng.osaka-u.ac.jp
}

\begin{abstract}
It is necessary to coordinate multiple tasks in order to cope with larger-scaled and more complicated tasks. However, it seems very hard to accomplish the multiple tasks at the same time. This paper proposes a method to resolve a conflict between task modules through the processes of their executions. Based on the proposed method, the robot can select an appropriate module according to the priority. In addition, we apply the module conflict resolution to a multiagent environment. Consequently, multiple tasks are automatically allocated to the multiple robots. As a task example, a soccer game is selected to show the validity of the proposed method. Real experiments are shown, and a discussion is given.
\end{abstract}

\section{Introduction}

In general, a robot is required to accomplish a variety of tasks in different environments. What is more important is to develop a method that can cope with multiple tasks at the same time. One approach to deal with such multiple tasks is to make some modules corresponding to the tasks. Each module has an ability to accomplish the corresponding task, that makes the programming easier. The most simple implementation is that one robot is allocated to the one task in advance. However, this approach seems inefficient from a viewpoint of the cost performance. We should not increase the number of robots even though the number of tasks increases.

Fontán and Matarić [3] proposed a method to divide a labor into exclusive spatial territories. That is, the working area is assigned to the robot. In order to realize their method, the knowledge about the working-field is given to the designer in advance.

Kuniyoshi [5] developed a framework called $\mathrm{Co}$ operation by Observation. Although they realized a number of cooperation in the real environment, the conflict between the modules should be considered in advance. Parker [7] proposed an architecture called $L$-ALLIANCE. Castelpietra et al [2] has shown a dynamic role (task) assignment method based on explicit communication. Although they realized some cooperation in the real environment, the number of the tasks for each robot is one. Therefore, the relationship between robots and tasks is exactly one to one correspondence.

This paper proposes a method to assign the multiple tasks to the multiple robots in a time-varying environment. Unlike previous work [2, 3, 7], we focus on the incompatibility of the modules called module conflict through the interactions with the environment by considering to what extent the executed module affects the accomplished modules.

The robot can discriminate the conflict modules between the current executed module and the accomplished module automatically. Based on the proposed method, the robot can accomplish the tasks as many as possible. The module conflict resolution is also applied to a multiple robots environment using explicit communication.

As a task example, a simplified soccer situation including multiple robots is introduced. Because there are several tasks to perform the soccer game, each robot has to do its own tasks in a dynamically changing environment. Based on the proposed method, two robots show the defending behaviors while they change the roles according to the situation. Real experiments are shown and a discussion is given.

\section{Dynamic Task Assignment}

\subsection{Definition of the Terminology}

For reader's understanding, we introduce some important terms. A multitask is a composition of multi- 

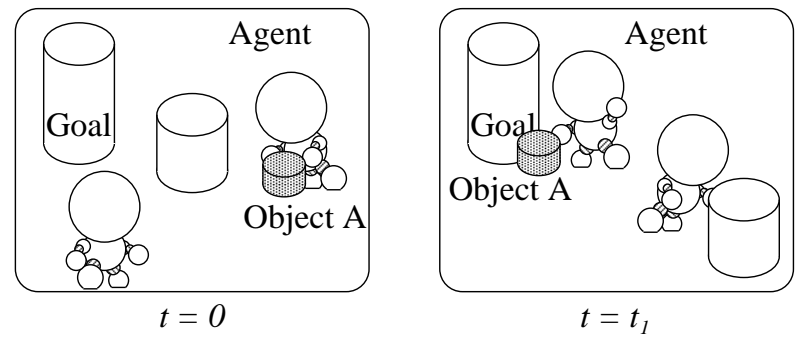

Figure 1: The definition of the task.

ple tasks, where a task means transfer from the initial state to the desired state by robot. A module consists of a policy (controller) and an evaluation function which indicates to what extent the current task is accomplished.

Figure 1 shows a simple example. There are two robots, one object (A), one obstacle, and, one goal (destination) in the environment. In this case, the multitask and the task are specified as follows:

multitask : To carry object A to the goal.

tasks : "Push object A", "Avoid an obstacle", "Approach an obstacle", "Push an obstacle", and so on.

\subsection{Module}

Figure 2 (a) shows a definition of a module. Each module $m_{k} \in \mathcal{M}$ has two functions. One is a policy $f_{k}(\boldsymbol{x})$ which maps from a state $\boldsymbol{x}$ to an action $\boldsymbol{o}_{k}$, and the other is an evaluation function $v_{k}(\boldsymbol{x})$. The module outputs $\boldsymbol{o}_{k}$ based on $f_{k}(\boldsymbol{x})$ in order to minimize the evaluation function $v_{k}(\boldsymbol{x})$. The module can be regarded as an actor-critic architecture [8]. In addition, we standardize $v_{k}(\boldsymbol{x})$ by

$$
a_{k}= \begin{cases}0 & \left(v_{k, \text { inf }}<v_{k}(\boldsymbol{x})\right) \\ \frac{v_{k}(\boldsymbol{x})-v_{k, \text { inf }}}{v_{k, \text { sup }}-v_{k, \text { inf }}} & \left(v_{k, \text { sup }}<v_{k}(\boldsymbol{x}) \leq v_{k, \text { inf }}\right) \\ 1 & \left(0 \leq v_{k}(\boldsymbol{x})<v_{k, \text { sup }}\right)\end{cases}
$$

where $v_{k, \text { inf }}$ and $v_{k, \text { sup }}$ are thresholds. Hereafter, we call $a_{k}$ task accomplishment $\left(0 \leq a_{k} \leq 1\right)$. Using the task accomplishment, the subset of modules $\mathcal{M}_{a}$ is defined by

$$
\mathcal{M}_{a}=\left\{m_{i} \mid a_{k}=1\right\} .
$$

A module $m \in \mathcal{M}_{a}$ is no longer executed because it has been already accomplished. In this sense, some

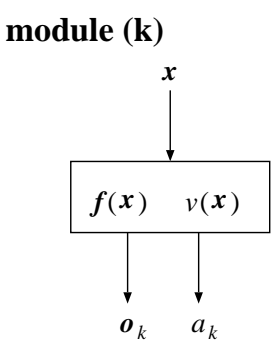

(a) module

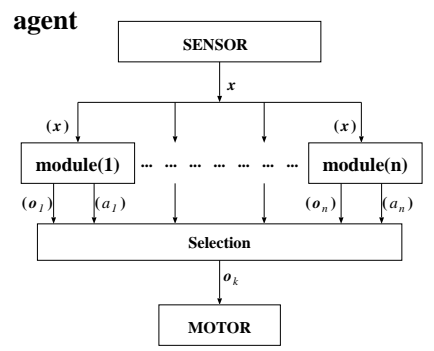

(b) agent
Figure 2: An architecture of the proposed method.

tasks might have been accomplished even if the corresponding modules have never been executed.

We define a new measure $e_{i}$ by

$$
e_{i}(t)=\left\{\begin{array}{cl}
1 & \text { if } m_{i} \text { is a selected module } \\
a_{i}(t) & \text { otherwise }
\end{array}\right.
$$

Hereafter, $e_{i}$ is called task execution, which is a scalar value between 0 and 1 . A case of $e_{i}=1$ and $a_{i} \neq 0$ means that the robot can not minimize the evaluation function although the robot attempt to accomplish the $i$-th task. To deal with time-varying environment, the averaged task execution is calculated by

$$
\bar{e}_{i}(t)=\rho \bar{e}_{i}(t-1)+(1-\rho) e_{i}(t),
$$

where $\rho$ is a forgetting factor between 0 and 1 .

\subsection{Module conflict resolution}

Although it is desirable for a robot to accomplish given multiple tasks, there would be some modules incompatible to each other according to the situation. Therefore, it is important for a robot to resolve such conflicts between the modules automatically.

In order to detect the conflict between the modules $m_{i}$ and $m_{j}$, a correlation of $e_{i}$ and $a_{j}$ is utilized. $\Delta r\left(e_{i}, a_{j}\right)$ is an index to measure how task execution of $m_{i}$ influences task accomplishment of $m_{j}$. The modules that are in conflict with the module $m_{j} \in \mathcal{M}_{a}$ is determined by

$$
\mathcal{M}_{c}=\left\{m_{i} \mid \Delta r\left(e_{i}, a_{j}\right)<0, \quad m_{j} \in \mathcal{M}_{a}\right\} .
$$

In case of $\Delta r\left(e_{i}, a_{j}\right) \geq 0$, the robot can cope with both the execution of the module $m_{i}$ and the accomplishment of the module $m_{j}$. On the other hand, in case of $\Delta r\left(e_{i}, a_{j}\right)<0$, the execution of the module $m_{i}$ prevents the accomplishment of the module $m_{j}$. 
The rest of the modules is determined as

$$
\mathcal{M}_{c a}=\mathcal{M}-\mathcal{M}_{a}-\mathcal{M}_{c}
$$

At the beginning, $\mathcal{M}_{c a}=\mathcal{M}, \mathcal{M}_{a}=\phi$, and $\mathcal{M}_{c}=\phi$. The robot selects the module by

$$
m_{s}=\arg \max _{m^{\prime} \in \mathcal{M}_{c a}} U\left(m^{\prime}\right)
$$

where $U$ is a priority which is given in advance. $\mathcal{M}_{a}$, $\mathcal{M}_{c}$ and $\mathcal{M}_{c a}$ are updated according to the changes of the environment in order to accomplish the tasks as many as possible.

Each module outputs an action based on the policy. The problem is how the robot should integrate the several outputs from the modules. Simple realization is based on the weighted sum of outputs. However, the resultant behavior is not guaranteed as an optimal one, and it may lead to some serious situations. Then, we introduce a priority function $U$ in order to make the priority. The robot follow the procedures every time step:

1. For the all modules, task accomplishment and task execution are computed using equations (1) and (2).

2. Calculate subsets $\mathcal{M}_{a}, \mathcal{M}_{c}$, and $\mathcal{M}_{c a}$.

3. Select a module $m_{s}$ from $\mathcal{M}_{c a}$ using equation (4).

4. Execute an action $\boldsymbol{o}_{s}$ based on the policy of the module $m_{s}$.

Because the actual outputs of the robot is one of the outputs, the behavior of the robot has a physical meaning for the selected module.

\section{Extension to a Multiagent Environ- ment}

As described in the previous section, we proposed a method to detect conflicts between modules. However, the modules belonging to $\mathcal{M}_{c}$ remain un-executed in the current system. Therefore, we extend our method to a multiagent system by adding multiple robots that take charge of $\mathcal{M}_{c}$. We assume that

- each robot has the same set of modules $\mathcal{M}$.

- each robot can get the information about task accomplishment and task execution.

This can be implemented by a blackboard system. Each robot has its own parameters such as task accomplishment, task execution, and module subsets $\left(\mathcal{M}_{a}\right.$, $\mathcal{M}_{c}$, and $\left.\mathcal{M}_{c a}\right)$. Hereafter, " $k$ " denotes the parameter of the $k$-th robot ${ }^{k} R$. In order to measure how much the robot ${ }^{k} R$ works, the load ${ }^{k} L_{j}(t)$ is calculated by

$$
{ }^{k} L_{j}(t)=\sum_{i=j}^{n}{ }^{k} a_{i} U\left(m_{i}\right),
$$

where $U\left(m_{i}\right)$ is the priority of module $m_{i}$.

For the current module $m_{j}$ determined by Eq. (4), the robot ${ }^{k} R$ judges whether $m_{j}$ should be executed or not. A procedure is described as follows:

1. Check task accomplishment of $m_{j}$.

Go to step 4 if no robot has already accomplished the task.

2. Check the remaining power of ${ }^{k} R$. If ${ }^{k} L(t)<L_{\max }$, go to step 4 ( $L_{\max }$ is a threshold).

3. Entrust the module $m_{j}$ to the other robot Subtract $m_{j}$ from ${ }^{k} \mathcal{M}_{c a}$, and add $m_{j}$ to ${ }^{k} \mathcal{M}_{e}$, where ${ }^{k} \mathcal{M}_{e}$ denotes the subset of the modules that are accepted by other robots. Then, return to step 1 .

4. Check the module conflict.

Go to step 5 if the modules $m_{j}$ and $m_{i}$ are compatible each other. Otherwise, add $m_{j}$ to ${ }^{k} \mathcal{M}_{c}$. Then, go to step 1.

5. Execute $m_{j}$.

If the robot $R_{k}$ accomplished the module $m_{j}$, transfer $m_{j}$ from ${ }^{k} \mathcal{M}_{c}$ to ${ }^{k} \mathcal{M}_{a}$. Then, go to step 1. Otherwise, execute $m_{j}$.

Each robot ${ }^{k} R$ computes the module conflict and obtains the subsets ${ }^{k} \mathcal{M}_{a},{ }^{k} \mathcal{M}_{c},{ }^{k} \mathcal{M}_{c a}$, and ${ }^{k} \mathcal{M}_{e}$, separately.

\section{Task and Assumptions}

\subsection{Environment and Robots}

We apply the proposed method to a simplified soccer game including two mobile robots in the context of RoboCup [4]. RoboCup is an increasingly successful attempt to promote the full integration of AI and robotics research, and many researchers around the world have been attacking a wide range of research issues. Here, the multitask for the robots is to defend the own goal.

A mobile robot has an omnidirectional vision system. The robot moves around the field based on the 


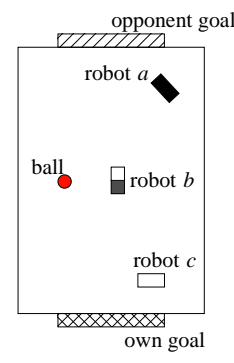

(a) top view of the field

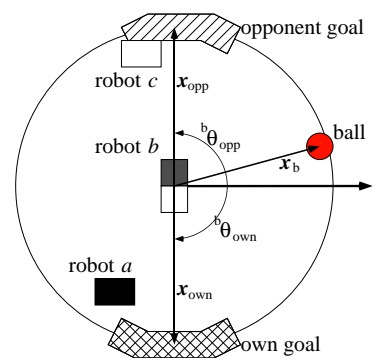

(b) view captured by an omnidirectional vision
Figure 3: The experimental setting.

power wheeled steering system. As motor commands, each robot has two degrees of freedom. The environment consists of a ball and two goals. The sizes of the ball and the goals are the same as those of the middlesize real robot league in the RoboCup Competition.

\subsection{Module description}

Figure 3 (b) shows detected image features to extract the information of the environment, where $\boldsymbol{x}_{b}$, $\boldsymbol{x}_{\text {own }}$ and $\boldsymbol{x}_{\text {opp }}$ are the center positions of the ball, the own goal, and the opponent goal, respectively. The policy and the evaluation function are designed based on these features. Figure 4 shows typical behaviors generated by the four prepared modules. In order to realize the defending behavior, we design the following modules.

$m_{1}$ : The purpose of this module is to push the ball.

$$
\begin{aligned}
\boldsymbol{x}_{d} & =\boldsymbol{x}_{b}, \\
v & =\left\|\boldsymbol{x}_{b}\right\|,
\end{aligned}
$$

where $\boldsymbol{x}_{d}$ and $\|\cdot\|$ denote the desired state and a norm of the vector, respectively. This module can be used when the robot attempts to shoot the ball into the goal or clear the ball.

$m_{2}$ : The purpose of this module is to move to the opposite side of the opponent goal.

$$
\begin{aligned}
\boldsymbol{x}_{d} & =(b+1) \boldsymbol{x}_{b}-b \boldsymbol{x}_{\text {opp }} \quad(0 \leq b \leq 1), \\
v_{r} & =\left\|\boldsymbol{x}_{b}\right\|, \\
v_{\theta} & ={ }^{b} \theta_{\text {opp }}, \\
v & =w v_{r}+(1-w) v_{\theta}, \quad(0 \leq w \leq 1),
\end{aligned}
$$

This module can be used when the robot behave as a offensive player.

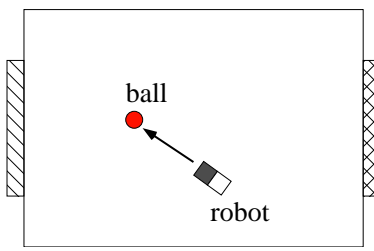

(a) $m_{1}$

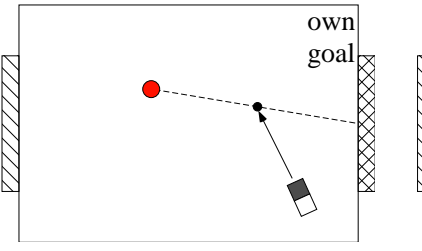

(c) $m_{3}$

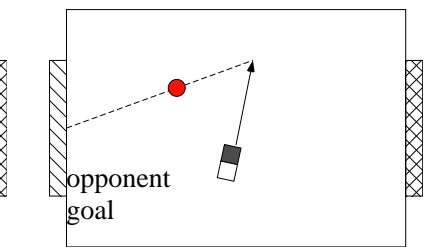

(b) $m_{2}$

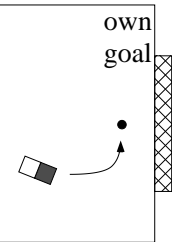

(d) $m_{4}$
Figure 4: Typical behaviors generated by the modules

$m_{3}$ : The purpose of this module is to move to the position between the ball and the own goal to save the own goal.

$$
\begin{aligned}
\boldsymbol{x}_{d} & =(1-a) \boldsymbol{x}_{b}+a \boldsymbol{x}_{\text {own }}, \\
v & =\pi / 2-\left|{ }^{b} \theta_{\text {own }}\right|,
\end{aligned}
$$

$m_{4}$ : The purpose of this module is to stay at the own goal.

$$
\begin{aligned}
\boldsymbol{x}_{d} & =\boldsymbol{x}_{\text {own }} \\
v & =\left\|\boldsymbol{x}_{\text {own }}\right\|,
\end{aligned}
$$

The goalie should select this module in order to block the own goal with its own body.

In addition, we define the priority as $U\left(m_{i}\right)=i$ in this experiment. We prepare a controller which makes the features on the image plane converge to the desired values. For the desired state $\boldsymbol{x}_{d}=\left[x_{d} y_{d}\right]^{T}$, a motor command $\boldsymbol{u}$ is computed by

$$
\boldsymbol{u}=\left[\begin{array}{l}
u_{r} \\
u_{l}
\end{array}\right]=\left[\begin{array}{cc}
-1 & 1 \\
1 & 1
\end{array}\right]\left[\begin{array}{l}
x_{d} \\
y_{d}
\end{array}\right],
$$

where $u_{r}$ and $u_{l}$ are the velocities of the right and left wheels, respectively. All the policies of the modules are designed using Eq. (6). Although we implement the above policies using the servoing technique in this experiment, it is possible to acquire them based on the learning algorithms [9] as well.

\section{Experimental Results}

We show a result to demonstrate how the proposed method works. Figure 5 shows a configuration of the 


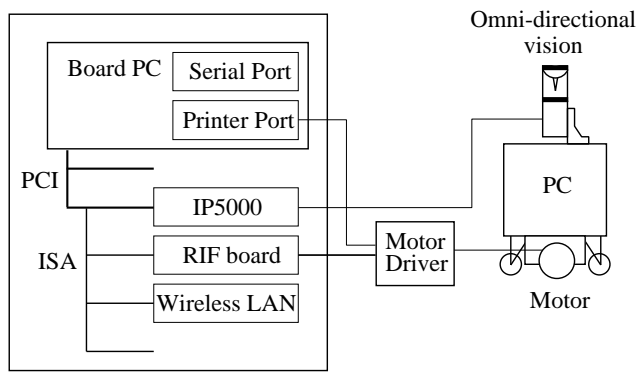

Figure 5: An overview of the robot system

real mobile robot. A simple color image processor (Hitachi IP5000) is applied to detect the ball and the goal area in the image in real-time $(33[\mathrm{msec}])$. Communication between the robots are realized using the Wireless LAN. Since the bandwidth of communication is very low, the robot can be controlled in real-time.

Figure 6 shows the averaged task accomplishment and task execution calculated by Eq. (3). From the beginning, $m_{4}$ is accomplished $\left(a_{4}=1\right)$, while r0 seldom executes $m_{1}$. On the other hand, according to the situation, $\mathrm{r} 1$ pushed the ball toward the opponent goal using the module $m_{1}$.

Figure 7 shows an example sequence of the robots' behaviors. For the sake of convenience, the robot colored in black (white) is called r0 (r1). In Figure 7 (a), $\mathrm{r} 0$ is the robot in the right side. From these figures,

(a), (b) : Both robots moved to the own goal because the module $m_{4}$ (stay at the own goal) is the most important.

(c) : Since r0 accomplished the module $m_{4}, \mathrm{r} 1$ neglected $m_{4}$ and checked the task accomplishment of $a_{i}(i=1,2,3)$.

(d) : r0 stayed in front of the own goal while r1 attempted to clear the ball.

(e) : They swapped their roles.

(f), (g) : r0 attempted to push the ball by $m_{1}$. In this case, $m_{2}$ was also accomplished by $\mathrm{r} 0$. On the other hand, r1 went to the own goal. However r1 failed to accomplish $m_{1}$ due to its poor policy.

(h) : r0 moved to the own goal again because r1 could not reach the own goal. As a result, r1 attempted to clear the ball again since $\mathrm{r} 1$ was not needed to stay at the own goal any more.

Because of the low image resolution, the robot sometimes failed to detect the objects at a long distance. In this case, the module could not be performed appropriately. In spite of those troubles, both robots were complementary to each other. The defense behavior could be accomplished based on our method.

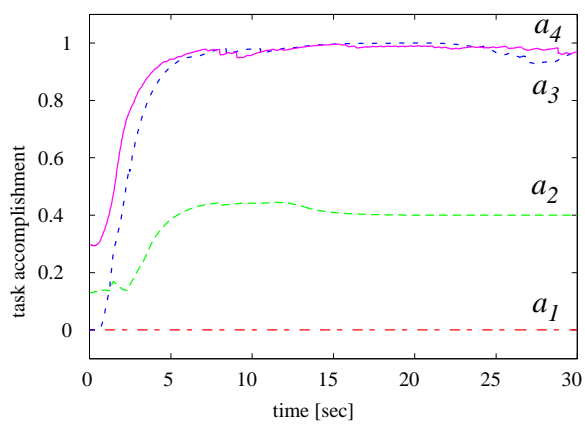

(a) r0's accomplishment

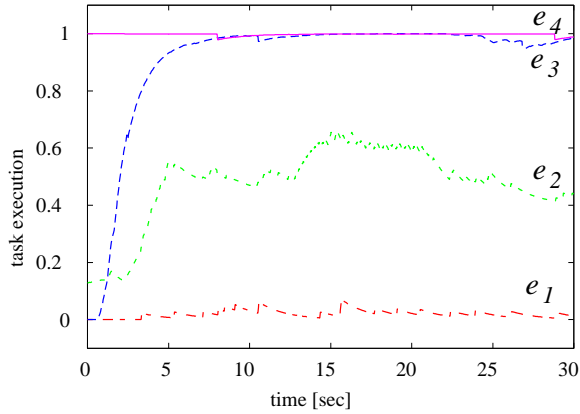

(b) r0's execution

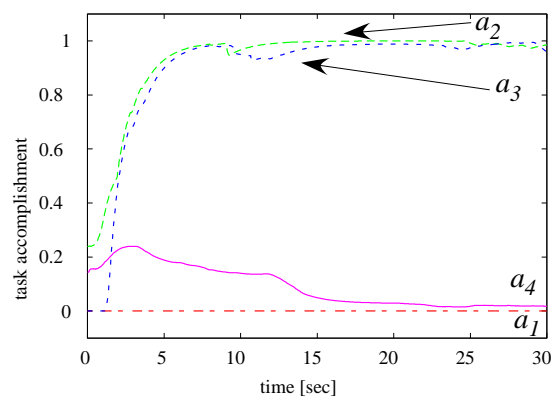

(c) r1's accomplishment

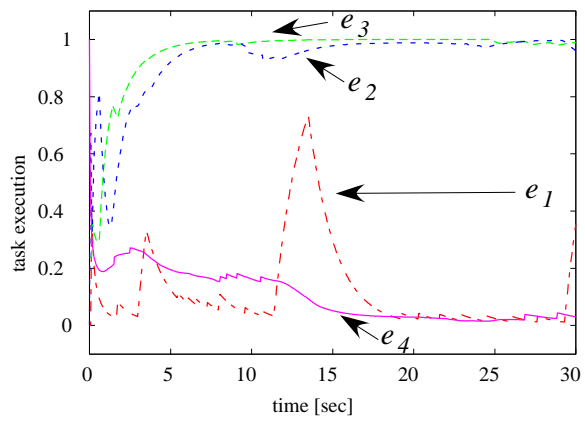

(d) r1's execution

Figure 6: The averaged task accomplishment $\overline{a_{i}}$ and task execution $\overline{e_{i}}$ 


\section{Conclusion}

We have proposed a method to resolve the conflict between the given modules to handle the multiple tasks in a multiagent environment. We have applied the proposed method to several simplified soccer games, and show the validity of our method in a dynamically changing environment. We have already checked the simulation results in case of three robots, and we are planning to perform the experiments using three mobile robots in the real environment.

As future work there are following issues. One is a study about the priority function $U$ because the total performance depends on the given priority function in the current system. Now, we are developing the method to change the priority function using the value of evaluation function. The other is an implementation of the proposed method without explicit communication since behavior understanding is also an important issue [6]. We have developed some algorithms to estimate the other's behavior from sequences of perception and action [1]. The integration of them leads the proposed method to be scaled to larger and more complicated situations.

\section{Acknowledgment}

This research was supported by the Japan Society for the Promotion of Science, in Research for the Future Program titled Cooperative Distributed Vision for Dynamic Three Dimensional Scene Understanding (JSPSRFTF96P00501).

\section{References}

[1] M. Asada, E. Uchibe, and K. Hosoda. Cooperative behavior acquisition for mobile robots in dynamically changing real worlds via vision-based reinforcement learning and development. Artificial Intelligence, 110:275-292, 1999.

[2] C. Castelpietra et al. Communication and Coordination among heterogeneous Mid-size players: ART99. In The Fourth International Workshop on RoboCup, pages 149-158, 2000.

[3] M. S. Fontán and M. J. Matarić. Territorial multirobot task division. IEEE Transaction on Robotics and Automation, 14(5):815-822, 1998.

[4] H. Kitano, ed. RoboCup-97 : Robot Soccer World Cup I. Springer Verlag, 1997.

[5] Y. Kuniyoshi. Behavior Matching by Observation for Multi-Robot Cooperation. In International Symposium of Robotics Research, 1995.
[6] T. Matsuyama. Cooperative Distributed Vision - Dynamic Integration of Visual Perception, Action, and Communication -. In Proc. of Image Understanding Workshop, Monterey CA, 111998.

[7] L. E. Parker. Lifelong Adaptation in Heterogeneous Multi-Robot Teams: Response to Continual Variation in Individual Robot Performance. Autonomous Robots, 8:239-267, 2000.

[8] R. S. Sutton and A. G. Barto. Reinforcement Learning. MIT Press/Bradford Books, March 1998.

[9] S. Suzuki, T. Kato, M. Asada, and K. Hosoda. Behavior Learning for a Mobile Robot with Omnidirectional Vision Enhanced by an Active Zoom Mechanism. In Proc. of Intelligent Autonomous System 5(IAS-5), pages 242-249, 1998.

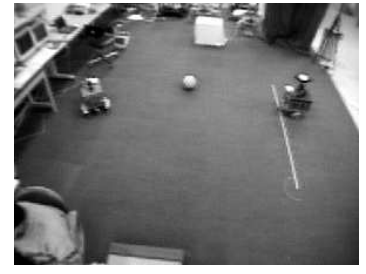

(a) Start

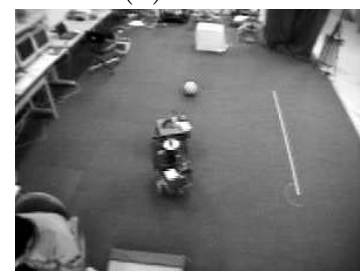

(c)

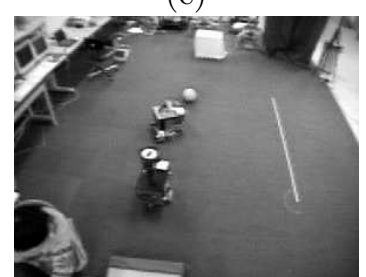

(e) $\mathrm{r} 0: 400$

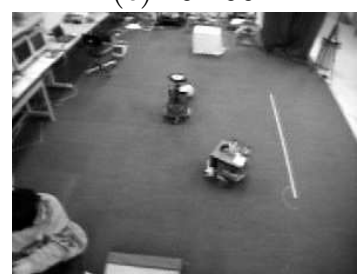

(g) $\mathrm{r} 1: 800$

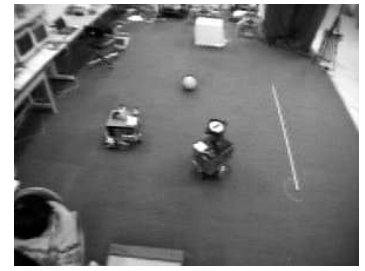

(b)

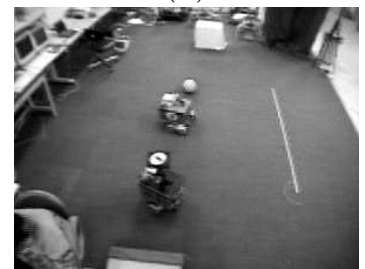

(d) $\mathrm{r} 1: 450$

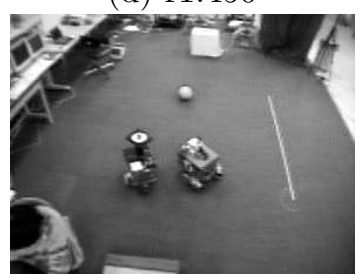

(f) r1:600

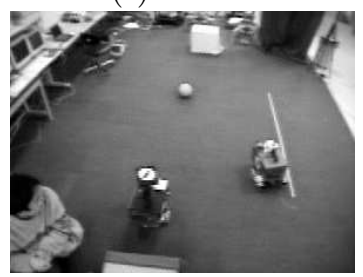

(h) $\mathrm{r} 0: \mathrm{t}=600$
Figure 7: The result of real space experiment (robot ID:time) 